\title{
Is strain elastography beneficial for isoechoic cholangiocarcinomas?
}

\author{
Veysel Burulday, Ural Koc, Sinan Tan
}

Kırıkkale University, Radiology Department, Kırıkkale, Turkey

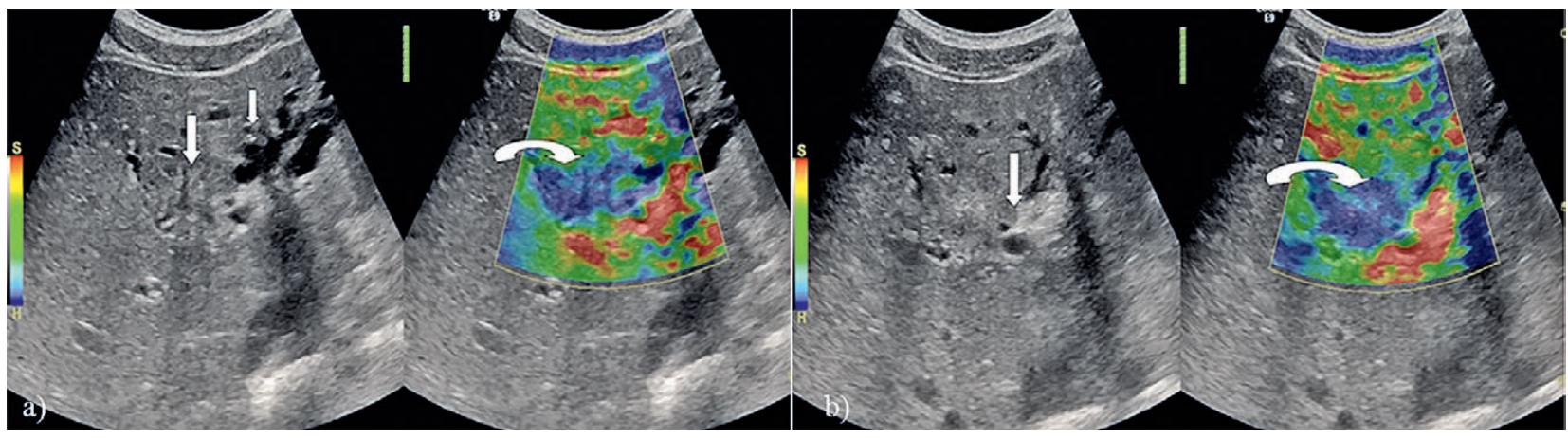

Fig 1. a) Ultrasonography revealed dilation in intrahepatic bile ducts, more prominent in the left side (short arrow) raising the suspicion of a isoechoic hilar cholangiocarcinoma (long arrow). Strain elastrography was encoded as hard lesion on the color scale (curved arrow); b) A remarkable interruption sign at the level of main hepatic bile duct (arrow) and hard lesion on the color scale (curved arrow) at strain elastrography were found.

\section{To the Editor,}

Ultrasonographic (US) aspect of hilar cholangiocarcinomas $(\mathrm{CCs})$ is isoechoic comparing to the liver parenchyma in $10 \%$ of cases and the diagnosis is established by visualizing dilation of intrahepatic bile ducts and sudden interruption of the bile duct caused by the mass lesion $[1,2]$.

A 30-year-old male patient was referred for the investigation of the etiology of jaundice. His physical examination showed no findings other than the jaundice observed on inspection. His medical history revealed no drug use. In laboratory tests total and direct bilirubin levels tended to be elevated with increased

Received 15.08.2016 Accepted 30.08.2016 Med Ultrason

2016, Vol. 18, No 4, 524-525, DOI: $10.11152 / \mathrm{mu}-884$

Corresponding author: Ural KOC, MD.

Kırıkkale University Faculty of Medicine

Department of Radiology

Kırıkkale, Turkey

Phone: 00905433672166

E-mail : dr_uralkoc@hotmail.com levels of aspartate aminotransferase, alanine aminotransferase, alkaline phosphatase, and gama-glutamyl transpeptidase. Hepatitis markers and HIV test results were negative. US revealed dilation of the intrahepatic bile ducts, more prominent in the left lobe. There was a remarkable interruption sign at the level of main hepatic bile duct. US findings raised the suspicion of a hilar CC with isoechoic appearance to the parenchyma. The patient underwent strain elastrography which showed more prominent lesion compared to the gray scale, which was encoded as hard lesion on the color scale (fig 1). These findings were confirmed by cross-sectional computed tomography and magnetic resonance images.

In recent years, there is a growing number of publications on elastographic assessment of focal liver lesions [3]. Onur et al [4] suggested that benign solid live lesions could be differentiated easily and noninvasively from malignant lesions using compression elastography. The values of mean strain index were higher compared to the other liver lesions, although the subtypes of lesions and the level of statistical significance were not specified for five patients with CCs 
evaluated in their study [4]. In another study, the lesion was detected using strain elastography before biopsy procedure and sampling was performed from the area encoded as hard lesion [5]. This finding can be mentioned as another advantage of strain elastography in patients with isoechoic CC allowing correct positioning for sampling during biopsy in addition to its contribution in detecting lesions. Guibal et al [6] reported that $\mathrm{CC}$ lesions were harder and with large variation of the homogeneity comparing to other lesions included in the study. Fibrotic components are known to exist in the histology of CCs [7]. Therefore, strain index and strain ratios are higher than the other focal liver lesions [3-5]. However, strain elastography can be beneficial in patients with isoechoic CC lesions; indeed, this method also allowed a clear visualization of the lesion in our case.

\section{References}

1. Bloom CM, Langer B, Wilson SR. Role of US in the detection, characterization, and staging of cholangiocarcinoma. Radiographics 1999;19:1199-1218.

2. Hann LE, Greatrex KV, Bach AM, Fong Y, Blumgart LH. Cholangiocarcinoma at the hepatic hilus: sonographic findings. AJR Am J Roentgenol 1997;168:985-989.

3. Bhargava S, Bhargava SK, Sharma S, Prakash M. Elastography: A new imaging technique and its application. JIMSA 2013;26:25-30.

4. Onur MR, Poyraz AK, Ucak EE, Bozgeyik Z, Özercan IH, Ogur E. Semiquantitative strain elastography of liver masses. J Ultrasound Med 2012;31:1061-1067.

5. Sandulescu L, Padureanu V, Dumitrescu C, et al. A pilot study of real time elastography in the differentiation of focal liver lesions. Curr Health Sci J 2012;38:32-35.

6. Guibal A, Boularan C, Bruce M, et al. Evaluation of shearwave elastography for the characterization of focal liver lesions on ultrasound. Eur Radiol 2013;23:1138-1149.

7. Lim JH. Cholangiocarcinoma: morphologic classification according to growth patern and imaging findings. AJR Am J Roentgenol 2003;181:819-827.

\section{Evaluation of nasal fluid flow in fetuses by Doppler ultrasound}

\section{Rafal Zielinski ${ }^{1}$, Maria Respondek-Liberska ${ }^{2}$}

${ }^{1}$ Department of Pediatric Otolaryngology, ${ }^{2}$ Department of Diagnostics and Prevention of Congenital Malformations, Polish Mother's Memorial Hospital - Research Institute, Medical University of Lodz, Lodz, Poland

\section{To the Editor,}

Ultrasonographic (US) assessment of fetal head and neck is usually performed in order to detect structural abnormalities [1]. Nevertheless, the use of color and spectral Doppler techniques allow better evaluation of fetal swallowing and fluid flow in the airways. Fetal

Received 12.08.2016 Accepted 31.08.2016

Med Ultrason

2016, Vol. 18, No 4, 525-526, DOI: 10.11152/mu-885

Corresponding author: Rafal Zielinski

Department of Pediatric Otolaryngology,

Medical University of Lodz

36/50 Sporna street, 91-738 Lodz, Poland

Phone/fax: +48-426177720

E-mail:rafal.zielinski@umed.lodz.pl breathing movements resulting in fluid flow in the upper respiratory tract, including the nose usually confirm proper growth and maturing of the lower respiratory tract [2].

Suzuki et al suggested that nasal fluid flow examination by US with spectral Doppler assessment might be important for evaluating fetal respiratory tract functions. They also found that a regular pattern of fast fetal nasal fluid flow was characteristic in the $3^{\text {rd }}$ trimester of gestation in normal fetuses [3].

So far parameters like fetal weight, fetal growth, fetal movements and amniotic fluid index based on prenatal ultrasound are considered as significant predictors of possible poor pregnancy outcomes [4,5]. Nevertheless, any other features of examination helping in the predic- 


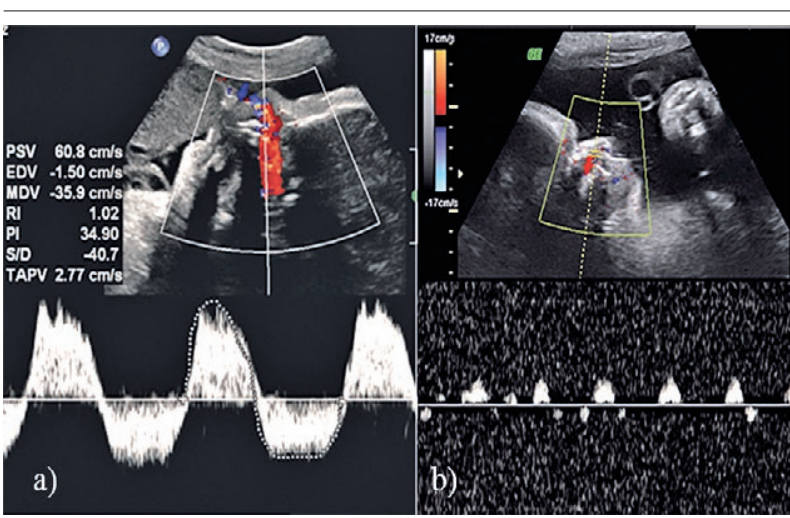

Fig 1. a) Fetus at $37^{\text {th }}$ week of gestation. No anomalies based on ultrasonography and echocardiography. Regular bidirectional fluid flow at the nose level with high velocities in both directions by two-dimensional Doppler ultrasound in sagittal plane. Vaginal delivery at 40th week of gestation. Newborn in good clinical condition; b) Fetus at $35^{\text {th }}$ week and 6 days of gestation. No structural anomalies based on ultrasonography and echocardiography. Nasal fluid flow by two-dimensional Doppler ultrasound in sagittal plane. Irregular nasal flow spectrum, with low velocities in both directions. Premature spontaneous delivery in the following 3 days. Suspicion of pneumonia based on the chest X-ray on the first day of postnatal life, CRP 50 $\mathrm{mg} / \mathrm{l}$, CPAP for 3 days, 7 days of iv antibiotic therapy, max. bilirubin level of $14 \mathrm{mg} \%$ and total hospital stay after delivery for 20 days.

tion of the course of pregnancy, perinatal period, and after birth condition might add additional value to the prenatal US assessment. Images of abnormal oral and nasal fluid flow and their qualitative assessment could possibly be helpful in detecting other than structural fetal abnormalities.

The full development of fetal behaviors related to regular breathing develops in the second half of pregnancy [6] and might be confirmed in $3^{\text {rd }}$ trimester, before the delivery. We do believe that the presence of pathological patterns of nasal amniotic fluid flows could serve as additional prognostic factor in otherwise normal fetuses by ultrasound (fig 1).
The prenatal examination of the upper respiratory system may provide information not only on head and neck morphological abnormalities but might serve as well as an additional tool informing about general fetus condition. Observation of fetal nasal breathing movements could be thus an additional US finding for confirming a good fetal condition with regular breathing movements and for detecting "abnormalities" in fetuses with irregular nasal breathing. In case of lack of fetal nasal breathing the prenatal detection of the very rare ventilation disorders (i.e. Ondine syndrome) could also be possible [7]. Therefore we believe that our observations could be valuable for the sonographers and sonologists as a new and important challenge.

\section{References}

1. Zielinski R, Respondek-Liberska M. [Craniofacial malformations in prenatal ultrasound evaluation. Literature review]. Ginekol Pol 2013;84:801-806.

2. Badalian SS, Chao CR, Fox HE .Timor-Tritsch IE. Fetal breathing-related nasal fluid flow velocity in uncomplicated pregnancies. Am J Obstet Gynecol 1993;169:563-567.

3. Suzuki M, Saito H, Yanaihara T. Assessment of fetal nasal fluid flow by two-dimensional color Doppler ultrasonography during pregnancy. J Matern Fetal Med 1999;8:159-163.

4. Smith-Bindman R, Chu PW, Ecker JL, Feldstein VA, Filly RA, Bacchetti P. US evaluation of fetal growth: prediction of neonatal outcomes. Radiology 2002;223:153-161.

5. Chousawai S, Tongprasert F, Yanase Y, Udomwan P. Tongsong $\mathrm{T}$. The efficacy of rapid biophysical profile in predicting poor pregnancy outcomes in suspected intrauterine growth restriction fetuses: preliminary study. J Med Assoc Thai 2012;95:482-486.

6. Nyberg MK, Johnsen SL, Rasmussen S, Kiserud T. Fetal breathing is associated with increased umbilical blood flow. Ultrasound Obstet Gynecol 2010;36:718-723.

7. Nannapaneni R, Behari S, Todd NV, Mendelow AD. Retracing "Ondine's curse". Neurosurgery 2005;57:354-363. 


\title{
Contrast-enhanced ultrasonography is useful for evaluating the intraplaque neovascularization in aortic complex plaque of ischemic stroke patients
}

\author{
Maiko Kouchi ${ }^{1}$, Atsushi Mizuma', Shizuka Netsu', Mai Ishihara ${ }^{2}$, Eiichiro Nagata', \\ Shunya Takizawa ${ }^{1}$
}

${ }^{1}$ Department of Neurology, ${ }^{2}$ Department of Medical Laboratory, Tokai University Hospital, Isehara, Kanagawa, Japan

To the Editor,

Intraplaque neovascularization is considered as a high-risk factor for embolism [1] and is correlated with plaque instability [2]. Contrast-enhanced ultrasonography (CEUS) has been used to evaluate intraplaque neovascularization of carotid artery plaque lesions $[3,4]$. However, we believe that the present report is the first to describe the use of CEUS to evaluate the neovascularization of complex aortic plaque lesions.

Ten acute ischemic stroke patients, in whom complex plaque lesions [5] on the aortic arch had been detected by transesophageal echocardiography (TEE), were recruited (median age 76 years [interquartile range (IQR); 65-80], 5 females). This study was approved by Tokai University Ethics Committee (13R-118), and written informed consent was obtained. To evaluate aortic plaque neovascularization, perflurobutane microbubbles were used as a contrast agent. TEE was done with an ARTIDA (Toshiba, Japan) equipped with a $4.4 \mathrm{MHz}$ multiplane probe. CEUS was performed in the coded phase inversion mode with mechanical index set at 0.2-0.3, depth 5 $\mathrm{cm}$, focus $3 \mathrm{~cm}$, harmonic frame 6.2 , frame rate 19 , and acoustic power $4 \%$. Perflurobutane $(0.01 \mathrm{ml} / \mathrm{kg})$ was injected intravenously as a bolus. About 5 minutes after the injection, we checked inflow of perflurobutane microbubbles in the aorticplaque. Inflammatory markers (high

Received 13.08.2016 Accepted 30.08.2016

Med Ultrason

2016, Vol. 18, No 4, 527-528, DOI: 10.11152/mu-890

Corresponding author: Atsushi Mizuma, MD, PhD

Department of Neurology, Tokai University

School of Medicine

143 Shimokasuya, Isehara, Kanagawa

259-1193, Japan

Phone: +81-463-1121, Fax: +81-463-1938

E-mail: atushi.mizuma@gmail.com

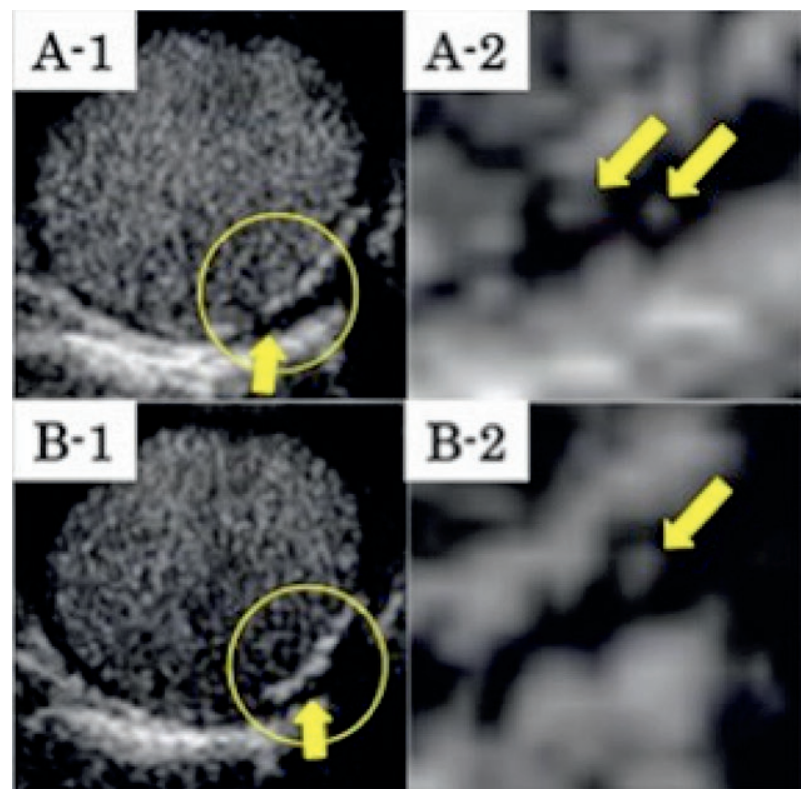

Fig 1. Perfluorobutane contrast-enhanced ultrasound imaging by transesophageal echocardiography. A-1, B-1: Microbubbles in aortic plaque of two patients at 5 min after intravenous injection of perfluorobutane $(0.01 \mathrm{ml} / \mathrm{kg})$; A-2, B-2: Enlarged images of enhanced lesions in aortic plaque. Moving bright spots within aortic plaque are indicated by arrows.

sensitivity CRP [hs-CRP] and IL-6) were checked in all patients. Six patients (enhanced group) showed moving bright spots within the plaque (fig 1). Hs-CRP was significantly higher in the enhanced group $(15,425 \mathrm{ng} / \mathrm{ml}$ [IQR; 5070-26900] vs $2565 \mathrm{ng} / \mathrm{ml}$ [101-1570], $\mathrm{p}<0.05$ ), although there was no significant difference of IL-6. High inflammatory markers in the enhanced group might reflect greater instability of complex plaque lesions [6]. We conclude that perflurobutane CEUS is a useful tool for evaluation of high-risk intraplaque neovascularization of complex plaque lesions on the aortic arch. 


\section{References}

1. Mathiesen EB, Bonaa KH, Joakimsen O. Echolucent plaques are associated with high risk of ischemic cerebrovascular events in carotid stenosis: the Tromso study. Circulation 2001;103:2171-2175.

2. Carlier S, Kakadiaris IA, Dib N, et al. Vasa vasorum imaging: a new window to the clinical detection of vulnerable atherosclerotic plaques. Curr Atheroscler Rep 2005;7:164-169.

3. Coli S, Magnoni M, Sangiorgi G, et al. Contast-enhanced ultrasound imaging of intraplaque neovascularization in carotid arteries: correlation with histology and plaque echogenicity. J Am Coll Cardiol 2008;52: 223-230.
4. Matsumoto N, Kimura K, Uno M, Sakai K, Sadahira Y, Nishimura $\mathrm{H}$. Enhanced carotid plaque on contrast-enhanced ultrasound is associated with plaque instability and rupture. Int J Stroke 2012;7:E12.

5. The Joint Committee of "The Japan Academy of Neurosonology" and "The Japan Society of Embolus Detection and Treatment" Guideline for Neurosonology. Exploration for Embolic Sources by Transesophageal Echo Cardiography. Neurosonology 2006;19:132-146.

6. Koenig W, Khuseyinova N. Biomarkers of atherosclerotic plaque instability and rupture. Arterioscler Thromb Vasc Biol 2007;27:15-26.

\title{
How to get ductus venosus flow velocity waveforms between 11 and 14 weeks: Candle Flame and Falling Drop Signs
}

\author{
Cemil Gürses
}

Department of Radiology Antalya Education and Research Hospital Antalya, Turkey

\section{To the Editor,}

Ductus venosus (DV) flow velocity waveforms are crucial for the early diagnosis of fetuses with chromosomal or heart and great arteries abnormalities [1].

For the accurate Doppler assessment of the DV some criteria are needed to be met and the competence in Doppler assessment of the DV is achieved only after extensive supervised training [2]. In order to achieve optimal DV waveform, color Doppler (CD) guiding is necessary and the pulsed Doppler (PD) sample gate should be placed in the correct place without contamination from the conjoined vessels.

Maiz et al suggested placing the gate in the yellowish aliasing area during fetal quiescence. However, it is often difficult due to the blooming artefact of the $\mathrm{CD}$ in spite of

Received 28.08.2016 Accepted 18.09.2016

Med Ultrason

2016, Vol. 18, No 4, 528-529, DOI: 10.11152/mu-886

Corresponding author: Cemil Gürses

Department of Radiology, Antalya Education \& Research Hospital

Antalya Eğitim ve Araştırma Hastanesi, Varlık Mah. Kazım Karabekir Cad. Soğuksu 7100 Antalya / Türkiye

Phone: +905066014545, Fax: +902422494462

E-mail: cemilgurses@hotmail.com the optimal CD preset parameters. Also, the fetal quiescence does not take long time when the US examinations start, therefore DV assessments should be performed as soon as possible. This requires the detailed visualization of the DV quickly. These can be circumvented by the integration of the DV morphology in electron microscopy (EM) scanning and the CD imaging findings.

In EM, there is a sphincter mechanism described as the shelf, the lip or the rim at the entrance to the DV in different studies [3]. The DV inlet is narrow due to 6-7 cell-layers consisting of abundant collagenous tissue, predominantly elastin [3]. The progressive widening is following the initial narrow segment (INS) and these give rise an hourglass appearance in the DV [3].

The hourglass in EM appears as a "candle flame" especially with advanced dynamic flow (ADF) CD imaging, which is a wide-band Doppler technique (WBDT) (fig 1a). If the Doppler sample gate is placed in the inner zone of the "flame" of the candle (thick white arrow in the figures), the correct PD flow velocity waveform is achieved easily. The solid wax of the candle represents the UV, the wick symbolizes the INS and the flame shows the progressive widening of the DV. Sometimes only the flame of the candle is visible without the solid wax, which characterizes the UV and the portal sinus. The flame without the solid wax can be likened to the "falling drop" (fig 1b). 


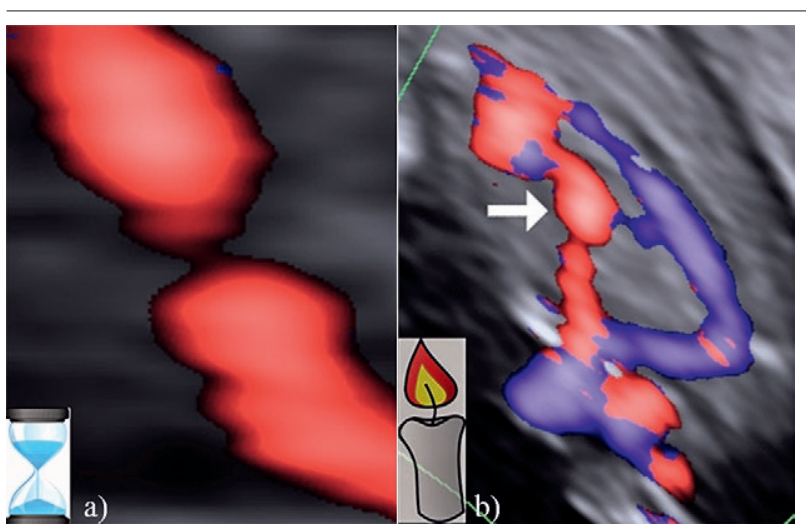

Fig 1. Resemblance between the candle flame (a) and the hourglass (b) and ductus venosus in color Doppler imaging with the ADF technique.

A Toshiba Applio TUS-A500 with a 2-6 MHz broadband convex transducer was used for imaging by a health care professional, who obtained the certificate of competence from the Fetal Medicine Foundation for the assessment of DV flow. Imaging parameters were set as dynamic range (DR) 70, dynamic frequency (DF) 3.0, and color gains (CG) 30 or 40, color PRF 5, 9 and color filter (F) 4. Sweep speed was $2 \mathrm{~cm} / \mathrm{s}$. The images were obtained using a standard of care clinical protocol; however, informed consent was obtained.

In conclusion the main difficulty of the DV measurements in the first trimester of the pregnancy is visualizing the vessel with $\mathrm{CD}$ due to the blooming, which gives rise to incorrect sampling in $\mathrm{PD}$, and the close proximity of the veins in the fetus.

The candle flame and the falling drop signs are practical and memorable clues and may shorten the optimal Doppler assessment of the ductus venosus training period.

\section{References}

1. Matias A, Gomes C, Flack N, Montenegro N, Nicolaides $\mathrm{KH}$. Screening for chromosomal abnormalities at 10-14 weeks: the role of ductus venosus blood flow. Ultrasound Obstet Gynecol 1998;12:380-384.

2. Maiz N, Kagan KO, Milovanovic Z, Celik E, Nicolaides $\mathrm{KH}$. Learning curve for Doppler assessment of ductus venosus flow at $11+0$ to $13+6$ weeks' gestation. Ultrasound Obstet Gynecol 2008;31:503-506.

3. Mavrides E, Moscoso G, Carvalho JS, Campbell S, Thilaganathan $\mathrm{B}$. The human ductus venosus between 13 and 17 weeks of gestation: histological and morphometric studies. Ultrasound Obstet Gynecol 2002;19:39-46.

\title{
Detection of arterioportal shunt on long term follow-up of a patient diagnosed with liver abscess
}

\author{
Larisa Sandulescu ${ }^{1,2}$, Sergiu Marian Cazacu ${ }^{1,2}$, Madalina Vintila ${ }^{2}$, Sineta Firulescu ${ }^{3}$, Laura \\ Lucan $^{3}$, Sarmis Marian Sandulescu ${ }^{4}$, Stefan-Cristian Dinescu ${ }^{3}$
}

${ }^{1}$ Research Center of Gastroenterology and Hepatology, University of Medicine and Pharmacy, ${ }^{2}$ Department of Gastroenterology, Emergency County Hospital, ${ }^{3}$ Department of Internal Medicine, Emergency County Hospital, ${ }^{4}$ Surgery Department, Emergency County Hospital Craiova, Romania

\section{To the Editor,}

Liver abscess (LA) is a well-defined purulent inflammation of the liver tissue, caused by a variety of pathogens. Typical morphology on ultrasound (US) ex-

Received 28.09.2016 Accepted 23.10.2016

Med Ultrason

2016, Vol. 18, No 4, 529-530, DOI: 10.11152/mu-887

Corresponding author: Stefan-Cristian Dinescu

76 Păltiniş street, 200127, Craiova, Romania

Phone: +40786025217

E-mail: stefandinescu@yahoo.com amination consists of an encapsulated lesion with a hypoechoic or anechoic center, depending on the stage of the disease. Although clinical resolution occurs earlier, residual abscess detected on US follow-up can persist even after 2 years [1]. Vascular complications associated with liver abscesses comprise most frequently arterial and venous thrombosis, arterial pseudoaneurysm and arterioportal shunt (APS) being a very rare occurrence [2].

We present the case of a 63 year old patient, who referred to out clinic accusing pain in the right hypo- 


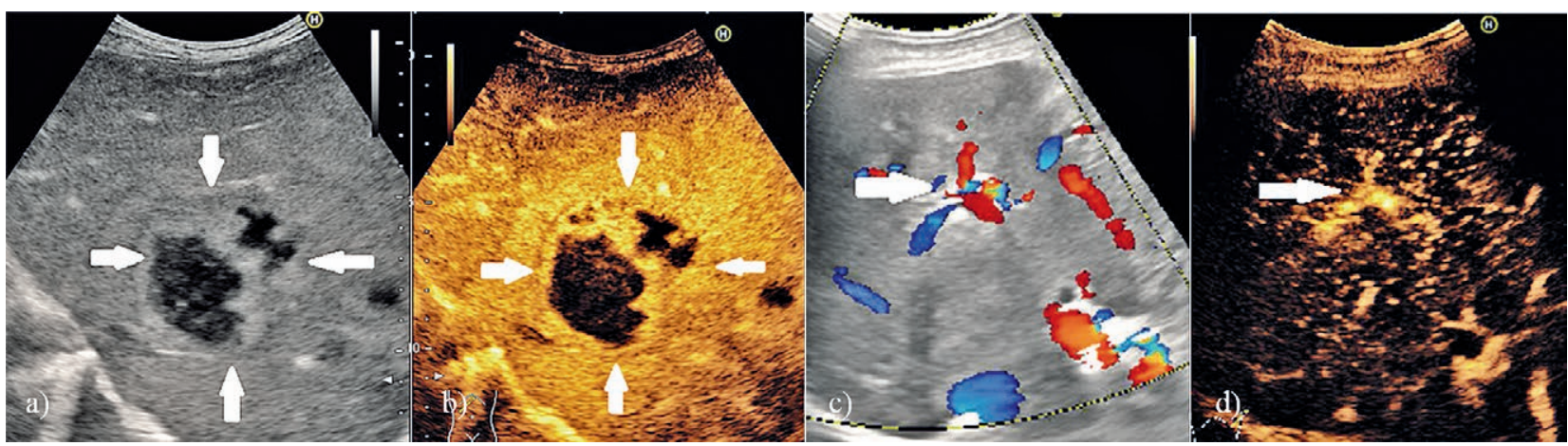

Fig 1. a) B-mode US morphology of liver abscess (arrows) on initial diagnosis; b) CEUS arterial phase showing typical incomplete peripheral enhancement (arrows) with enhancement of the septa (arrow head); c) and d) follow-up examination after 2 years; c) arterial blood flow pattern of the arterioportal shunt on color-Doppler mode (arrow); d) aspect of arterioportal shunt (arrow) on CEUS.

chondrium, weight loss, loss of appetite, and fatigue. Clinical examination revealed subicteric sclera and the laboratory test hinted to the presence of an inflammatory process (marked leukocytosis and elevated erythrocyte sedimentation rate); viral markers for hepatitis were absent and the tumor markers were within normal range. US examination revealed a well-defined oval mass in the right liver lobe (RLL), with hyperecogenic walls, hypoecogenic center and a few septa, with no vascularization on Doppler-mode (fig 1a). On contrast-enhanced ultrasound (CEUS) examination, the RLL formation displayed an incomplete, peripheral enhancement and enhancement of the septa in the arterial phase (fig 1b), with wash-out in the tardive phase, suggestive for liver abscess. After two year, at follow-up evaluation, a significant resolution of the LA was found. Doppler US examination revealed in the area near the residual LA a blood flow pattern with arterial and venous communication, suggestive of APS (fig 1c). CEUS showed a homogeneous enhancement of this region, indicated also the presence of the APS (fig 1d).

Involvement of the intrahepatic vascular system secondary to a LA consists mainly of portal and hepatic vein thrombosis [3]. Less common vascular abnormalities are hepatic artery pseudoaneurysm and APS. APS is defined as a functional or organic communication between the arterial branch and portal venous system, causing redistribution of the arterial flow into a focal region of the portal venous flow. This compensatory relationship between the two blood supply sources of the liver maintains the liver perfusion when vascular compromise occurs [4]. Diagnostic criteria of APS are based mainly on high frequency systolic Doppler shifts, resulting from a large pressure gradient between the artery and the recipient vein. On color Doppler imaging, these shunts display high-velocity, low-resistance arterial waveforms with arterialised portal venous flow [5]. Local inflammation and relatively frequent regional venous thrombosis associated with LA may be the main underlying mechanisms that explain this rare occurrence of APS in the present case.

\section{References}

1. K C S, Sharma D. Long-term follow-up of pyogenic liver abscess by ultrasound. Eur J Radiol 2010;74:195-198.

2. Kang M, Bapuraj JR, Khandelwal N, Kochhar R, Kalra $\mathrm{N}$, Verma GR. Liver abscess associated with hepatic artery pseudoaneurysm with arteriovenous fistula: imaging and interventional management. Acta radiol 2006;47:162-166.

3. Dutta A, Bandopadhyay S. Management of liver absces. Medicine Update 2012;9:1-7.

4. Ternberg JL, Butcher HR Jr. Blood flow relation between hepatic artery and portal vein. Science 1965;150:10301031.

5. Bhargava P, Vaidya S, Kolokythas O, Katz DS, Dighe M. Hepatic vascular shunts: Embryology and imaging appearances. Br J Radiol 2011;84:1142-1152. 


\title{
Sonographer preference for Knox versus ballistic gelatin for the creation of deep venous thrombosis ultrasound phantoms
}

\author{
Patrick Olivieri, Michael Doctor, Sebastian Siadecki, Gabriel Rose, Aaran Drake, \\ Turandot Saul
}

Department of Emergency Medicine, Emergency Ultrasound Division, Mount Sinai St. Luke's / Mount Sinai Roosevelt Hospital Center, New York, New York

\section{To the Editor,}

Simulation-based teaching can enhance learner confidence and the ability to perform various ultrasound scans for diagnosis and procedural guidance. Phantoms can be particularly useful in the demonstration of pathology, which may not always be readily available in a human model. Commercially available phantoms exist; however, their expense limits widespread use.

Recipes for homemade gelatin phantoms have been shown to be suitable for practice and for teaching a variety of sonographic applications [1-2]. A sonographic evaluation for deep venous thrombosis (DVT) involves the compression of the vessel as an indicator of vein patency. Many of the phantoms that follow traditional recipes using store-bought gelatin are too delicate for use in DVT education and puncture when pressure is applied. Ballistic gelatin closely simulates the density and viscosity of human tissue and is used as a standardized medium for testing the performance of firearms ammunition. It is used instead of muscle tissue, as its properties can be carefully controlled, allowing for consistent and reliable comparisons.

The specific aims of this study were to describe our process of creating DVT ultrasound phantoms using store-bought gelatin and commercial ballistic gelatin and

Received 20.10.2016 Accepted 02.11.2016

Med Ultrason

2016, Vol. 18, No 4, 531-532, DOI: 10.11152/mu-888

Corresponding author: Turandot Saul

Mount Sinai St. Luke's Mount Sinai West

Department of Emergency Medicine

1000 Tenth Avenue, Room G-01

New York, New York 10019

(c) $917-209-5110 /$ (f) 212-523-8000

E-mail: tsaul@chpnet.org

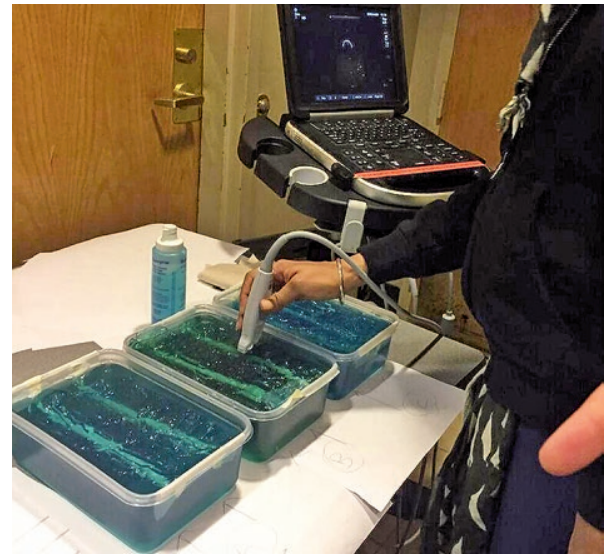

Fig 1. Ultrasound phantom containing compressible and non-compressible vessels

to compare their various sonographic features, durability and shelf life.

DVT phantoms were created by suspending waterfilled 5/8" Penrose drains (Convidien, Minneapolis, $\mathrm{MN}$ ) half way up the side of a plastic container. Pieces of a 0.28 " diameter hot glue gun stick (3M, St. Paul, $\mathrm{MN})$ were inserted into several areas of the Penrose drains to create areas of non-compressibility. The gelatin preparations described below were poured over the suspended Penrose drains.

Knox gelatin (Kraft Foods, Northfield, IL) is inexpensive ( $\$ 1.00$ /ounce) and is available at most supermarkets. A $10 \%$ concentration (three $0.25 \mathrm{oz}$ packets of gelatin/1 cup of water) was used to create the phantom. Ballistic gelatin is available from a number of manufactures and can be ordered online. 10lbs of ballistic gelatin powder (Vyse Gelatin Co, Schiller Park, IL) cost $\$ 125$ (\$0.78/ounce) plus $\$ 18$ for a de-foamer solution recommended for clarity by the manufacture. A $10 \%$ concentra- 
tion solution was used for the ballistics gelatin as well. Several drops of blue food coloring were added to make the models transluscent. Models were gently agitated for 60 seconds to remove air bubbles prior to solidification.

Twenty emergency medicine residents attended a 15-minute lecture on the basics of DVT ultrasound. Two Sonosite Edge systems (Bothell, WA) with L25 high frequency (13-6MHz) linear transducers were used to scan the phantoms (fig 1); then, residents completed a survey. The phantoms were covered with a plastic wrap, refrigerated and checked weekly. The Knox and ballistic gelatin powder cost $\$ 6.00$ and $\$ 5.08$ respectively to create one phantom. After the educational session, the Knox phantoms were cracked in multiple places and the ballistic phantom was intact. Mold growth was present on the Knox gelatin after 3 weeks of storage with no growth on the ballistic gelatin after 12 weeks. $18 / 20(90 \%)$ of residents stated that the ballistic gelatin allowed for clearer visualization of the vessel. 15/20 $(75 \%)$ said the Knox gelatin was more similar to human tissue in consistency and firmness, and 16/20 (80\%) concluded that the Knox gelatin had less distracting particles. The group was divided (10/20 [50\%]) each, for which product best returned to baseline after com- pression. Ballistic gelatin was favored over Knox gelatin in overall preference $(13 / 20[65 \%])$.

Ballistics gelatin was preferred by the educators for the following reasons: 1) creation of the models is time consuming and the more durable the gel, the less this process would have to be repeated; 2) ballistics gelatin is recyclable. It can be removed from its container and melted; 3) the cost per phantom of Knox gelatin is more than ballistic gelatin; 4) the ballistic gelatin was more durable after repeated compression.

Overall, the ballistic gelatin phantom was preferred by the emergency medicine residents for its sonographic features and it was preferred by the investigators because of its durability and longer shelf-life.

\section{References}

1. Bude RO, Adler RS. An easily made, low-cost, tissuelike ultrasound phantom material. J Clin Ultrasound 1995;23:271-273.

2. Jafri F, Runde D, Saul T, Lewiss RE. An inexpensive and easy simulation model of ocular ultrasound that mimics normal anatomy as well as abnormal ophthalmologic conditions. J Ultrasound Med 2011;30:569-573. 